\title{
Generation of maximally entangled states of qudits using twin photons
}

\author{
Leonardo Neves,${ }^{1}$ G. Lima, ${ }^{1}$ J. G. Aguirre Gómez,${ }^{1,2}$ C. H. Monken, ${ }^{1}$ C. Saavedra, ${ }^{2}$ and S. Pádua ${ }^{1, \text { Æ }}$ \\ ${ }^{1}$ Departamento de Física, Universidade Federal de Minas Gerais. Caixa Postal 702, Belo Horizonte, MG 30123-970, Brazil. \\ ${ }^{2}$ Center for Quantum Optics and Quantum Information, Departamento de Fisica, \\ Universidad de Concepción, Casilla 160-C, Concepción, Chile.
}

(Dated: July 31, 2021)

\begin{abstract}
We report an experiment to generate maximally entangled states of $\mathcal{D}$-dimensional quantum systems, qudits, by using transverse spatial correlations of two parametric down-converted photons. Apertures with $\mathcal{D}$-slits in the arms of the twin photons define the qudit space. By manipulating the pump beam correctly the twin photons will pass only by symmetrically opposite slits, generating entangled states between these different paths. Experimental results for qudits with $\mathcal{D}=4$ and 8 are shown. We demonstrate that the generated states are entangled states.
\end{abstract}

PACS numbers: 03.67.Mn, 03.65.Ud, 03.67.Hk

The interest in studying higher dimensional entangled states comes both from the foundations of quantum mechanics and from the development of new protocols in quantum communication. For instance, it was demonstrated that maximally entangled states of two quantum systems in a $\mathcal{D}$-dimensional Hilbert space, qudits, violate local realism stronger than qubits [1]. Entangled qudits are more resistant to noise than qubits, as was shown in [1, 2]. In quantum cryptography 3 , the use of entangled qutrits $(\mathcal{D}=3)[4,[5]$ or qudits $[\underline{6}, 7]$ instead of qubits is more secure against eavesdropping attacks. Moreover, one knows that the protocols like quantum teleportation [8] or quantum cryptography [3], work best for maximally entangled states. All these facts motivate the development of techniques to generate entangled states among quantum systems in higher dimensional Hilbert space with a good quality of entanglement.

Recently, spontaneous parametric down-conversion (SPDC) has been used for realizing entangled qudits. In Ref. 9] four polarization entangled photons are used to obtain two entangled qutrits. The use of two photons in higher dimensional space is another possibility. Entangled qutrits with two photons using an unbalanced 3-arm fiber optic interferometer 10] or photonic orbital angular momentum 11] has been demonstrated. Time-bin entangled qudits up to $\mathcal{D}=11$ from pump pulses generated by a mode-locked laser has also been reported [12].

In this letter, we demonstrate the experimental generation of maximally entangled states of qudits by using the transverse spatial correlations of the photon pairs (biphotons) produced by SPDC. Biphotons are sent through apertures with $\mathcal{D}$-slits. The $\mathcal{D}$ possible paths (slits) followed by each photon of the pair are defined as our qudit space. Due to a transference of information from the pump laser beam to the two-photon state 13], we can control the transverse correlations of the photon pairs passing by the slits, by manipulating the pump beam. A proper manipulation of this one allow us to make the biphotons pass only by symmetrically opposite slits, generating entangled states between these different trans- verse spatial modes. Results for qudits with $\mathcal{D}=4$ and 8 are shown and the scheme described here can be extended to higher dimensions. We give a brief theoretical description of this process and present the experimental results and discussion.

Here, it is sufficient to write the equations in one dimension. Considering the degenerate case and using the monochromatic, paraxial and thin crystal approximations, the two-photon state generated by SPDC and transmitted through apertures is given by 14

$$
\begin{aligned}
|\Psi\rangle= & \lambda \int d q_{1} \int d q_{2} \int d x_{1} \int d x_{2} e^{i \frac{k}{8 z_{A}}\left(x_{2}-x_{1}\right)^{2}} e^{-i\left(q_{1} x_{1}+q_{2} x_{2}\right)} \\
& \times A_{1}\left(x_{1}\right) A_{2}\left(x_{2}\right) W\left(\frac{1}{2}\left(x_{1}+x_{2}\right) ; z_{A}\right)\left|1 q_{1}\right\rangle\left|1 q_{2}\right\rangle,(1)
\end{aligned}
$$

where $q_{j}$ and $x_{j}$ are the transverse components of the wave vector and position, respectively, of the downconverted photons in modes $j=1,2 ; A_{j}\left(x_{j}\right)$ is the transmission function of the aperture in mode $j$ and $W\left(\xi ; z_{A}\right)$ is a function that describes the pump beam transverse field profile at a longitudinal distance $z_{A}$ from the crystal (plane of the apertures). We see that the pump beam transfers an information to the two-photon state. Defining the apertures, we can modify the transverse spatial correlations of the twin photons by modifying the function $W\left(\xi ; z_{A}\right)$, which is achieved by manipulating the pump beam.

Suppose that each photon component of the downconverted pair is sent to a $\mathcal{D}$-slit $(\mathcal{D} \geq 2)$ in $z=z_{A}$. The transmission function of this aperture is

$$
A_{j}\left(x_{j}\right)=\sum_{l=-l_{D}}^{l_{D}} \Pi\left(\frac{x_{j}-l d}{2 a}\right),
$$

where $j=1,2, l_{D} \equiv(\mathcal{D}-1) / 2$ and $\Pi(\xi)$ is a rectangle function; $a$ is the slit half width and $d$ is the separation between two consecutive slits. Notice that the index $l$ works as the label of a given slit displaced by $l d$ from the origin of the $\mathcal{D}$-slit aperture. Inserting Eq. (2) into Eq. (11) and considering a narrow pump beam transverse profile $W\left(\xi ; z_{A}\right)$, which is nonvanishing only inside 
a small interval $[-a, a]$, around the center $(\xi=0)$ of the plane $z=z_{A}$ (this can be achieved by focusing the pump beam), we can show that [14]

$$
|\Psi\rangle=\frac{1}{\sqrt{\mathcal{D}}} \sum_{l=-l_{D}}^{l_{D}} e^{i k \frac{d^{2} l^{2}}{2 z} A}|l\rangle_{1} \otimes|-l\rangle_{2}
$$

where

$$
|l\rangle_{j} \equiv \sqrt{\frac{a}{\pi}} \int d q_{j} e^{-i q_{j} l d} \operatorname{sinc}\left(q_{j} a\right)\left|1 q_{j}\right\rangle .
$$

The $\left\{|l\rangle_{j}\right\}$ states satisfy the condition ${ }_{j}\left\langle l \mid l^{\prime}\right\rangle_{j}=\delta_{l l^{\prime}}$ and represent the photon in mode $j$ transmitted by the slit $l$. We use them to define the logical states of the qudits. Therefore, the two-photon state in Eq. (3) has the form of a maximally entangled state of two qudits having correlations such that if the photon in mode 1 passes through the slit $l$ the photon in mode 2 will pass through the symmetrically opposite slit $-l$. These correlations satisfy the transverse momentum conservation in the thin crystal approximation [13]. The relative phases are due to the optical path length difference of the biphoton from the crystal to a given pair of slits.

With the experimental setup outlined in Fig. 1 we make measurements for $\mathcal{D}=4$ and 8. A 5-mm-long BBO ( $\beta$-barium borate) crystal, cut for type-II phase matching is pumped by a $100 \mathrm{~mW}$, frequency-doubled Ti: sapphire laser operating at $413 \mathrm{~nm}$. Down-converted photons with a degenerate wavelength $\lambda=826 \mathrm{~nm}$ are produced at an angle of $2.5^{\circ}$ off the pump direction. Two identical $\mathcal{D}$ slits $\left(A_{1}\right.$ and $\left.A_{2}\right)$ are placed at the exit path of the downconverted beams at the same distance $z_{A}=200 \mathrm{~mm}$ from the crystal. The slit width is $2 a=0.09 \mathrm{~mm}$ and the distance between two consecutive slits is $d=0.17 \mathrm{~mm}$. Single and coincidence counts are measured by detectors $D_{1}$ and $D_{2}$ placed behind the $\mathcal{D}$-slits at a distance $z \approx 2 \mathrm{~mm}$. In front of each detector there is a single slit of width $0.1 \mathrm{~mm}$ (oriented parallel to the slits of $A_{i}$ ), followed by an interference filter of $8 \mathrm{~nm}$ bandwidth, centered at $826 \mathrm{~nm}$. A lens of $f=250 \mathrm{~mm}$ is placed in the Gaussian pump beam at $50 \mathrm{~mm}$ before the crystal such that it is focused in the plane of the $\mathcal{D}$-slits, as shown in the inset of Fig. 1 This transverse profile ensures that the state in Eq. (3) will be formed after the $\mathcal{D}$-slits.

In order to identify this state we make a coincidence selective measurement onto the basis $\left\{|l\rangle_{1}\left|l^{\prime}\right\rangle_{2}\right\}$ as follows: detector $D_{1}$ is kept fixed behind the slit $l$ while detector $D_{2}$ is scanning the other $\mathcal{D}$-slit in the $x$ direction. After $\mathcal{D}$ measurements like this $\left(D_{1}\right.$ ranging from $l=\frac{-(\mathcal{D}-1)}{2}$ to $l=\frac{\mathcal{D}-1}{2}$ ) we will have the probability amplitudes for all $\mathcal{D}^{2}$ basis states $\left\{|l\rangle_{1}\left|l^{\prime}\right\rangle_{2}\right\}$. According to the quantum state in Eq. (3) each such measurement will select only $|l\rangle_{1}|-l\rangle_{2}$. Thus, it is expected that coincidences occur only when the detector $D_{2}$ scan the slit $l^{\prime}=-l$. No diffraction or interference is observed in this process, because the detectors are very close to the slits (Fig. (1).

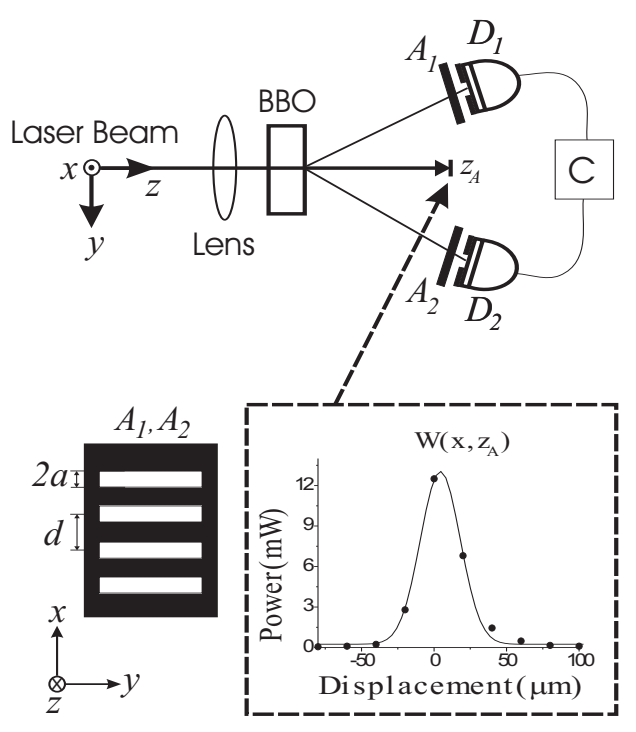

FIG. 1: Outline of the experimental setup. The inset shows the transverse profile of the pump beam in the $\mathcal{D}$-slits plane, used in the measurements.

The results for $\mathcal{D}=4$ and $\mathcal{D}=8$ are shown in Figs. 2 and 3. respectively. One can see that the experimental data are in agreement to what we describe above: there are coincidence peaks only when the detector $D_{2}$ scan the slit symmetrically opposite to that which detector $D_{1}$ is fixed. It is also noteworthy that these peaks have approximately the same number of coincidences within the error bars. This means that the states $|l\rangle_{1}|-l\rangle_{2}$ will have the same amplitudes. To see this we calculate the probabilities for all basis states. These ones are defined as $P_{l_{1} l_{2}^{\prime}}=C_{l_{1} l_{2}^{\prime}} / \sum_{\left\{l_{1}, l_{2}^{\prime}\right\}} C_{\left\{l_{1} l_{2}^{\prime}\right\}}$, where $C_{l_{1} l_{2}^{\prime}}$ is the number of coincidences between detector $D_{1}$ at slit $l$ and $D_{2}$ at $l^{\prime}$ 15. Figure 2(e) shows a histogram of probabilities for all basis states of $\mathcal{D}=4$. We clearly observe there, a structure of 4-dimensional maximally entangled qudits. We observe the same for $\mathcal{D}=8$. So within the experimental errors the states $|l\rangle_{1}|-l\rangle_{2}$ are equally weighted. For $\mathcal{D}=4$ these errors are around $4 \%$ and we obtain

$$
\begin{aligned}
|\Psi\rangle= & 0,50\left|-\frac{1}{2},+\frac{1}{2}\right\rangle+0,50\left|+\frac{1}{2},-\frac{1}{2}\right\rangle \\
& +e^{i \frac{k d^{2}}{z_{A}}}\left(0,49\left|-\frac{3}{2},+\frac{3}{2}\right\rangle+0,49\left|+\frac{3}{2},-\frac{3}{2}\right\rangle\right),
\end{aligned}
$$

which has a fidelity $F=0.98 \pm 0.08$ to that state predicted by theory in Eq. (3). For $\mathcal{D}=8$, with errors around $3 \%$, we have

$$
\begin{aligned}
|\Psi\rangle= & 0,36\left|-\frac{1}{2},+\frac{1}{2}\right\rangle+0,34\left|+\frac{1}{2},-\frac{1}{2}\right\rangle \\
& +e^{i \frac{k d^{2}}{z_{A}}}\left(0,34\left|-\frac{3}{2},+\frac{3}{2}\right\rangle+0,34\left|+\frac{3}{2},-\frac{3}{2}\right\rangle\right) \\
& +e^{i \frac{3 k d^{2}}{z_{A}}}\left(0,34\left|-\frac{5}{2},+\frac{5}{2}\right\rangle+0,36\left|+\frac{5}{2},-\frac{5}{2}\right\rangle\right) \\
& +e^{i \frac{6 k d^{2}}{z_{A}}}\left(0,36\left|-\frac{7}{2},+\frac{7}{2}\right\rangle+0,35\left|+\frac{7}{2},-\frac{7}{2}\right\rangle\right),(6
\end{aligned}
$$

with a fidelity $F=0.96 \pm 0.05$ to that state in Eq. (3). Phases in Eqs. (5) and (6) were not measured. They are 
a)

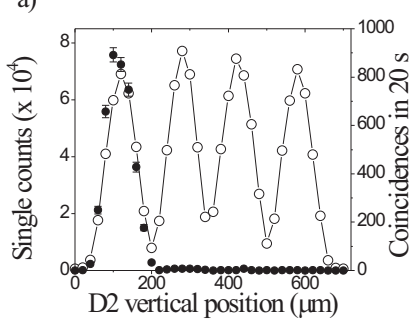

c)

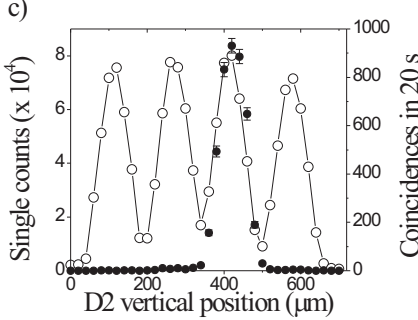

e)

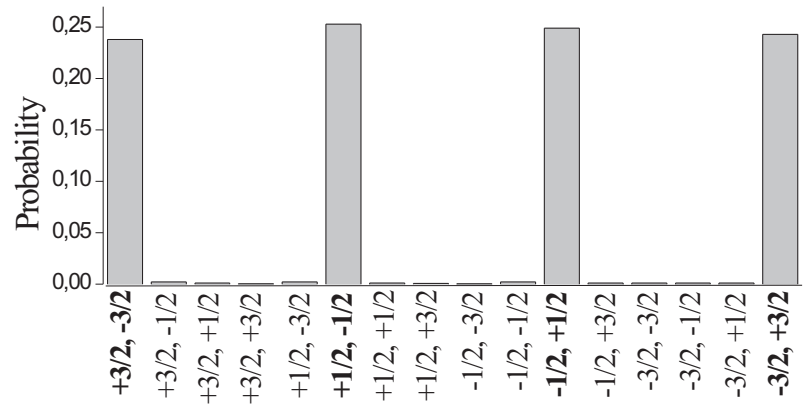

FIG. 2: $\mathcal{D}=4 . D_{2}$ single counts (०) and $D_{1}-D_{2}$ coincidence counts $(\bullet)$ measured simultaneously with $D_{1}$ fixed behind the slit $l$ and $D_{2}$ scanning in $x$ direction. From left to right the single count peaks are the slits $l^{\prime}=-\frac{3}{2}, \ldots,+\frac{3}{2} . D_{1}$ is fixed behind the slit $l$ (a) $+\frac{3}{2}$, (b) $+\frac{1}{2}$, (c) $-\frac{1}{2}$, and (d) $-\frac{3}{2}$. (e) Histogram of probabilities for all basis states $\left\{|l\rangle_{1}\left|l^{\prime}\right\rangle_{2}\right\}$.

dependant on fixed experimental parameters and they can be cancelled by choosing right values for $d$ and $z_{A}$ or by adding an appropriate external phase to a given slit.

Although the measurement procedure that we adopt demonstrates that the correlations between the two photons agree with the theoretical predictions, it is not sufficient to guarantee that we have a coherent superposition of product states $|l\rangle_{1}|-l\rangle_{2}$. Exactly the same results shown in Figs. 2 and 3 could be obtained by an equally weighted mixture like

$$
\rho_{\mathrm{cc}}=\frac{1}{D} \sum_{l=-l_{D}}^{l_{D}}|l\rangle_{11}\langle l|\otimes|-l\rangle_{22}\langle-l| .
$$

This state, also called classically correlated, exhibits the same pairwise correlation that one in Eq. (3). If in each measurement the detectors are selecting one pair of independent spatial modes, then we should have the state $\rho_{\text {cc }}$. Let us show that is not the case here, i.e., the prepared two-photon state after the $\mathcal{D}$-slit is indeed a pure a)

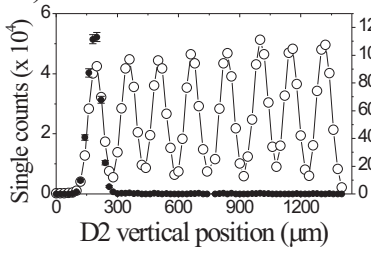

b)
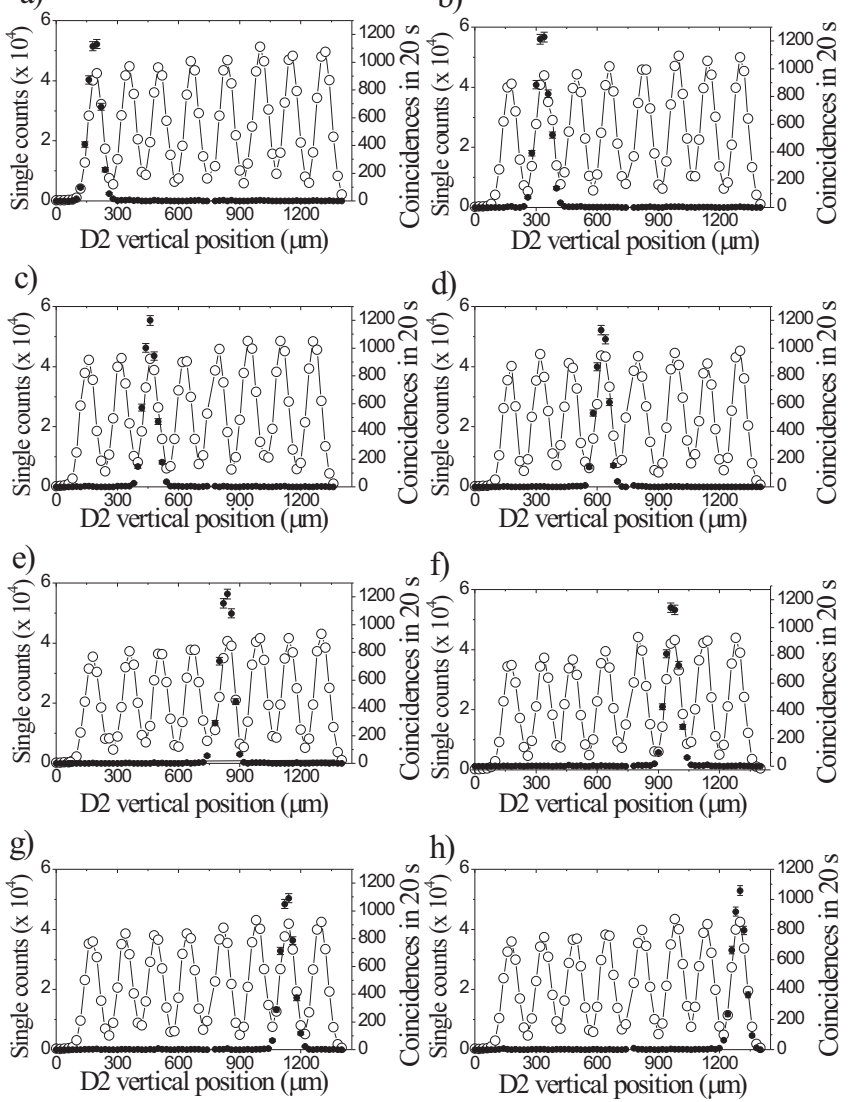

h)

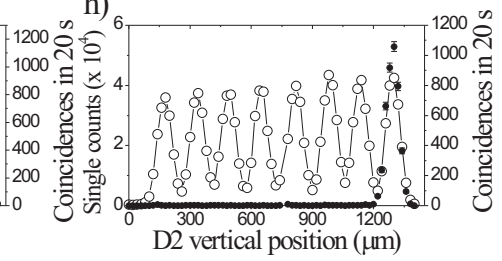

FIG. 3: $\mathcal{D}=8 . D_{2}$ single counts (o) and $D_{1}-D_{2}$ coincidence counts $(\bullet)$ measured simultaneously with $D_{1}$ fixed behind the slit $l$ and $D_{2}$ scanning in $x$ direction. From left to right the single count peaks are the slits $l^{\prime}=-\frac{7}{2}, \ldots,+\frac{7}{2} . D_{1}$ is fixed behind the slit $l$ (a) $+\frac{7}{2}$, (b) $+\frac{5}{2},(\mathrm{c})+\frac{3}{2},(\mathrm{~d})+\frac{1}{2},(\mathrm{e})-\frac{1}{2}$, (f) $-\frac{3}{2},(\mathrm{~g})-\frac{5}{2}$, and $(\mathrm{h})-\frac{7}{2}$.

and entangled state. We define $\mathbb{E}_{j} \equiv \hat{E}^{(+)}\left(x_{j}, z\right)$ where $\hat{E}^{(+)}\left(x_{j}, z\right)$ is the field operator for the mode $j$ and $x$ is the detection position in the plane $z$. So, the photodetection coincidence rate $C\left(x_{1}, x_{2}\right)$ is proportional to $\operatorname{Tr}\left(\rho \mathbb{E}_{1}^{\dagger} \mathbb{E}_{2}^{\dagger} \mathbb{E}_{2} \mathbb{E}_{1}\right)$ [16. We consider detectors placed at $z \gg z_{A}$ after the apertures (Fraunhofer regime). Experimentally, this can be achieved by inserting a convergent lens with focal length $f$, after the apertures, in each arm of the twin photons at a distance $z_{L}$ from the crystal. In this way, the electric field operator is 13,17

$$
\mathbb{E}=\int d q \int d q^{\prime} \hat{a}\left(q^{\prime}\right) e^{i\left(q x-q^{2} \frac{\left(z-z_{L}\right)}{2 k}-q^{\prime 2} \frac{\left(z-z_{\mathcal{A}}\right)}{2 k}+f \frac{\left(q-q^{\prime}\right)^{2}}{2 k}\right)} .
$$

The coincidence rate for the state in Eq. (3) will be

$$
\begin{aligned}
C\left(x_{1}, x_{2}\right) \propto & \sum_{l=-l_{D}}^{l_{D}} V_{l l}\left(x_{1}, x_{2}\right)+2 \sum_{l=-l_{D}}^{l_{D}-1} \sum_{m=l+1}^{l_{D}} V_{l m}\left(x_{1}, x_{2}\right) \\
& \times \cos \left(\beta(l-m)\left[x_{2}-x_{1}-(l+m) \phi\right]\right), \quad(8)
\end{aligned}
$$


where $\beta=k f d /\left[f^{2}-\left(z-z_{L}-f\right)\left(z-z_{A}-f\right)\right]$ and $\phi=d\left[f^{2}-\left(z-z_{L}-f\right)\left(z+z_{A}-f\right)\right] / 2 f z_{A} ; V_{l m}\left(x_{1}, x_{2}\right) \equiv$ $\prod_{(r=1,2)(s=l, m)} \operatorname{sinc}\left(a \beta\left[x_{r}+(-1)^{r} s \eta d\right] / d\right)$, are the single slit diffraction terms where $\operatorname{sinc}(x) \equiv(\sin x) / x$ and $\eta=$ $\left(z-z_{L}-f\right) / f$. For a classically correlated state $C\left(x_{1}, x_{2}\right)$ will be given only by the first sum in Eq. (8). We see that for an entangled state, the coincidence rate exhibits an interference pattern with conditional fringes [18] (the fringes depend on the position of a given detector) while for the mixture we will have only single slit diffraction and no interference. So, when we treat spatial correlations of two photons, the observation of a fourth-order interference pattern with conditional fringes is a sufficient signature for entanglement.

Fourth-order interference patterns were obtained for $\mathcal{D}=4$. With the same setup shown in Fig. 1 we put the detectors at $z=800 \mathrm{~mm}$ from the crystal and convergent lenses with focal length $f=150 \mathrm{~mm}$ were inserted in each arm of the twin photons at $z_{L}=650 \mathrm{~mm}$ from the crystal. Coincidence measurements were made as a function of $x$ position of the detector $D_{1}$ while $D_{2}$ is kept fixed. The results are shown in Fig. 固 (i) $D_{2}$ fixed at $x_{2}=0 \mu \mathrm{m}$

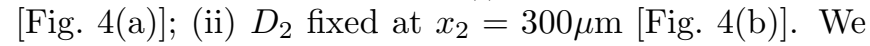
clearly observe a two-photon interference pattern with conditional fringes. The solid curves fitting the experimental data, are obtained from the theoretical expression in Eq. (8) with only one normalization parameter and taking into account the finite size of the detectors. The discrepancy between experimental data and theory is likely due to the small acceptance angle of the detectors. Nevertheless, the main result shown in Fig. U1 is the existence of a two-photon interference pattern which has conditional fringes. This agree with the coincidence rate in Eq. (8) and as we said, for spatially correlated photon pairs, this is a signature for entanglement. Therefore, as we wish demonstrate the two-photon state prepared in our experiment is a pure and entangled state of qudits with $\mathcal{D}=4$ and $\mathcal{D}=8$.

We have presented a scheme to generate maximally entangled states of qudits by using two photons produced by SPDC and exploring the transverse spatial correlation between them. The generated state is seen to have a high fidelity to that predicted by theory and we show also that it is indeed a pure and entangled state. This experiment could be used for tests on the foundations of quantum mechanics. In addition, there is also the possibility of carry out this experiment with the $\mathcal{D}$-slits replaced by optical fibers. This makes possible the construction of quantum-optical logic gates to achieve protocols in quantum communication that need higher dimensional entangled states.

We acknowledge S. P. Walborn and L. Davidovich for very useful discussions. This work was supported by the Brazilian agencies CAPES, CNPq, and MilênioInformação Quântica. Saavedra was supported by Grants Nos. FONDECYT 1010010 Milenio ICM P02-49F.
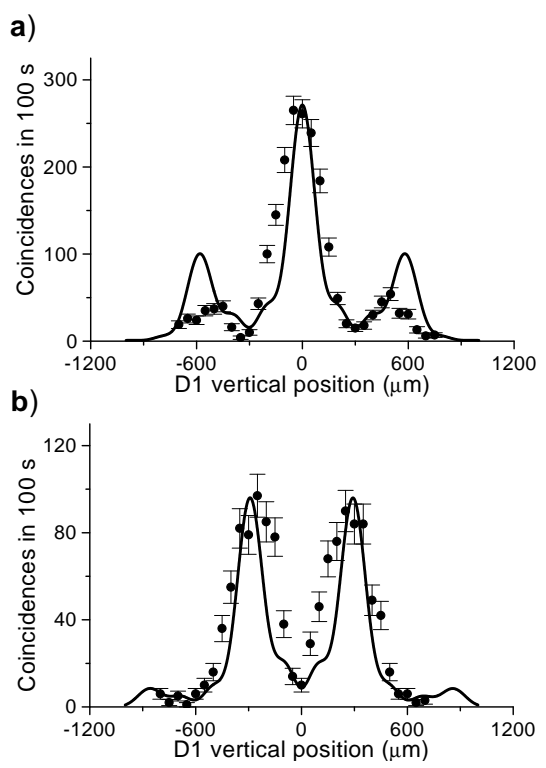

FIG. 4: Fourth-order interference patterns for two photons transmitted by 4 -slits as a function of $D_{1}$ position. (a) $D_{2}$ is kept fixed at $x_{2}=0 \mu \mathrm{m}$. (b) $D_{2}$ is kept fixed at $x_{2}=300 \mu \mathrm{m}$.

* Electronic address: spadua@fisica.ufmg.br

[1] D. Kaslikowski et al., Phys. Rev. Lett. 85, 4418 (2000).

[2] D. Collins et al., Phys. Rev. Lett. 88, 040404 (2002).

[3] A. K. Ekert, Phys. Rev. Lett. 67, 661, (1991).

[4] H. Bechmann-Pasquinucci and A. Peres, Phys. Rev. Lett. 85, 3313 (2000).

[5] T. Durt, N. J. Cerf, N. Gisin, and M. Zukowski, Phys. Rev. A 67, 012311 (2003).

[6] M. Bourennane, A. Karlsson, and G. Björk, Phys. Rev. A 64, 012306 (2001).

[7] N. J. Cerf, M. Bourennane, A. Karlsson, and N. Gisin, Phys. Rev. Lett. 88, 127902 (2002).

[8] C. H. Bennett et al., Phys. Rev. Lett. 70, 1895 (1993).

[9] J. C. Howell, A. Lamas-Linares, and D. Bouwmeester, Phys. Rev. Lett. 85, 030401 (2002).

[10] R. T. Thew, A. Acín, H. Zbinden and N. Gisin, Phys. Rev. Lett. 93, 010503 (2004).

[11] A. Vaziri, G. Weihs, and A. Zeilinger, Phys. Rev. Lett. 89, 240401 (2002).

[12] H. de Riedmatten, I. Marcikic, H. Zbinden and N. Gisin, Quant. Inf. and Comp. 2, 425 (2002).

[13] C. H. Monken, P. H. Souto Ribeiro, and S. Pádua, Phys. Rev. A 57, 3123 (1998).

[14] Leonardo Neves, S. Pádua, and Carlos Saavedra, Phys. Rev. A 69, 042305 (2004).

[15] Each $C_{l_{1} l_{2}^{\prime}}$ is the number of coincidences in four points of maximum single counts of the scanned slit $l^{\prime}$.

[16] M. O. Scully and M. S. Zubairy, Quantum Optics, Cambridge University Press, Cambridge (1997).

[17] Ivan F. Santos, M. A. Sagioro, C. H. Monken, and S. Pádua, Phys. Rev. A 67, 033812 (2003).

[18] D. M. Greenberger, M. A. Horne, and A. Zeilinger, Phys. Today 46 (8), 22 (1993). 